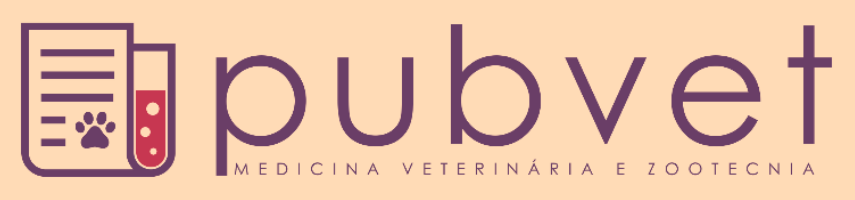

https://doi.org/10.31533/pubvet.v13n4a319.1-6

\title{
GnRH agonista (Acetato de histrelina) na múltipla ovulação em égua
}

\author{
Davy Aparecido Almeida Santos ${ }^{1 \oplus}$, Matheus Batista de Oliveira ${ }^{1^{*} \odot}$, Heder Nunes Ferreira $^{2,3} \Theta$ \\ ${ }^{1}$ Graduando em Medicina Veterinária, Faculdade Pio Décimo, Aracaju-SE, Brasil. \\ ${ }^{2}$ Médico Veterinário, Hospital Veterinário Dr. Vicente Borelli, Faculdade Pio Décimo, Aracaju-SE, Brasil. \\ ${ }^{3}$ Docente do curso de Medicina Veterinária, Faculdade Pio Décimo, Aracaju-SE, Brasil. \\ *Autor para correspondência: matheusoliveiravet@gmail.com
}

Resumo. Os equinos possuem particularidades fisiológicas reprodutivas, apresentando peculiaridades na morfofisiologia folicular. A manipulação farmacológica do ciclo estral na égua é rotineiramente utilizada, pelo fato desta apresentar grande variação na duração do estro. Para se obter altas taxas de concepção a deposição do sêmen no sistema reprodutivo da fêmea deve ser realizada próximo ao período ovulatório, com utilização de apenas uma dose inseminante. Por essa razão são utilizados fármacos como o acetato de deslorelina e histrelina acetato com finalidade de induzir a ovulação, assim como a múltipla ovulação. Objetivou-se descrever o efeito farmacológico do histrelina acetato para múltipla ovulação em égua. Foi utilizada uma égua, Quarto de Milha, com idade de cinco anos, situada em central de reprodução localizada na cidade de Nossa Senhora do Socorro-SE. Para a indução da múltipla ovulação foi administrado histrelina acetato na dose de $100 \mu \mathrm{g}$, realizando avaliação do sistema reprodutivo e acompanhamento do grau de edema uterino, diâmetro folicular e sua resposta ao fármaco indutor. Foram realizadas análises ultrassonográficas para identificação de diâmetro folicular, com posterior administração de prostaglandina (PGF2 $\alpha$ ) para promover luteólise do último ciclo. Para o segundo ciclo, foi administrado histrelina acetato $(100 \mu \mathrm{g} / \mathrm{BID})$ durante cinco dias. No sexto dia foi administrado o mesmo fármaco com a primeira dose diária de $(100 \mu \mathrm{g})$ e a segunda de $(250 \mu \mathrm{g})$ para induzir à múltipla ovulação. Conclui-se que o animal não respondeu ao protocolo estabelecido, acreditando ser devido à dose utilizada e pelo início da administração quando os folículos possuíam diâmetros superiores, não demonstrando o efeito farmacológico esperado.

Palavras-chave: dinâmica folicular, manipulação do estro, reprodução equina, superovulação

\section{GnRH agonist (Histrelin acetate) on multiple ovulation in mare}

Abstract. The horses have physiological reproductive particularies, presenting peculiarities in the follicular morphophysiology. The pharmacological manipulation of the estrous cycle in the mare is routinely used, since it presents high variation in the duration of estrus. In order to obtain higher conception rates, semen deposition in the female reproductive system should be performed close to the ovulatory period, with the use of only one inseminating dose. For this reason, drugs such as deslorelin acetate and histrelin acetate are used to induce ovulation, as well as multiple ovulations. The aim was to describe the pharmacological effect of histrelin acetate for multiple ovulations in mare. It was used a mare, Quarter Horse, five years old, located in a breeding center in the city of Nossa Senhora do Socorro-SE. For an oocyte ovulation induction was administred Histrelin acetate in the dose of $100 \mu \mathrm{g}$, performing evaluation of the reproductive system and monitoring the degree of endometrial edema, follicular diameter and its response to the inducing drug. Ultrasonographic analyzes were performed to identify follicular diameter, with subsequent administration of prostaglandin (PGF2 $\alpha$ ) to promote luteolysis of the last cycle. For the second cycle, histrelin acetate (100 $\mu \mathrm{g} / \mathrm{BID})$ was administered during five 
days. On the sixth day the same drug was administered with the first daily dose of (100 $\mu \mathrm{g})$ and the second dose $(250 \mu \mathrm{g})$ to induce multiple ovulations. It was concluded that the animal did not respond to the protocol established, believing it to be due to dose used and the start of administration when the follicles have with superior diameters, not demonstrating the expected pharmacological effect.

Key-words: equine reproduction, follicular dynamics, manipulation of estrus, superovulation

\section{GnRH agonista (Acetato de histrelina) en la múltiple ovulación en yegua}

Resumen. Los equinos poseen particularidades fisiológicas reproductivas, presentando peculiaridades en la morfofisiología folicular. La manipulación farmacológica del ciclo estral en la yegua es rutinariamente utilizada, por el hecho de esta presentar gran variación en la duración del estro. Para obtener altas tasas de concepción la deposición del semen en el sistema reproductivo de la hembra debe ser realizada cerca del período ovulatorio, con utilización de apenas una dosis inseminante. Por esta razón se utilizan fármacos como el acetato de deslorelina e histelina acetato con el fin de inducir la ovulación, así como la múltiple ovulación. Se objetivó describir el efecto farmacológico del histelina acetato para múltiple ovulación en la yegua. Se utilizó una yegua, Cuarto de Milla, con edad de cinco años, situada en la central de reproducción ubicada en la ciudad de Nuestra Señora del Socorro-SE. Para la inducción de la múltiple ovulación se administró histelina acetato con dosis de $100 \mu \mathrm{g}$, realizando evaluación del sistema reproductivo y seguimiento del grado de edema uterino, diámetro folicular y su respuesta al fármaco inductor. Se realizaron análisis ultrasonográficos para identificación de diámetro folicular, con posterior administración de prostaglandina (PGF2 $\alpha$ ) para promover luteólisis del último ciclo. Para el segundo ciclo, se administró histelina acetato (100 $\mu \mathrm{g}$ / BID) durante cinco días. En el sexto día se administró el mismo fármaco con la primera dosis diaria de $(100 \mu \mathrm{g})$ y la segunda de $(250 \mu \mathrm{g})$ para inducir a la múltiple ovulación. Se concluye que el animal no respondió al protocolo establecido, creyendo ser debido a la dosis utilizada y al inicio de la administración cuando los folículos tenían diámetros superiores, no demostrando el efecto farmacológico esperado.

Palabras claves: dinámica folicular, manipulación del estro, reproducción equina, superovulación

\section{Introdução}

A reprodução é um dos principais fatores que tem impulsionado o cenário da equideoculutura no Brasil e no mundo, favorecendo ao aprofundamento em pesquisas com biotecnologias que promovam à eficiência nos sistemas produtivos, a exemplo da manipulação do ciclo estral. As características do ciclo estral são variáveis entre os animais domésticos, particularmente os equinos, devido à singular fisiologia reprodutiva desta espécie (Ginther et al., 2003; Ginther et al., 1972). Essa peculiaridade culmina na ampliação de biotécnicas reprodutiva, como o uso de fármacos para a manipulação do ciclo estral.

Existem diversos índices reprodutivos que permitem avaliar a eficiência de um sistema de criação, destacando-se a taxa de concepção. Esta pode ser otimizada e obter valores elevados a partir da deposição do sêmen no trato reprodutivo da égua próximo à ovulação (Woods et al., 1990).

O uso de fármacos indutores de ovulação é realizado há décadas (Loy \& Hughes, 1966), sobretudo por uma necessidade constante do retorno econômico da atividade, obtendo-se mais animais durante uma estação reprodutiva. A indústria farmacêutica veterinária desenvolveu fármacos que permitem que a sincronização do estro e tempo para ovulação pré-definido, minimizando as avaliações reprodutivas exageradamente, além da mão de obra necessária. A indução de ovulação é recomendada quando há uma limitação do sêmen a ser utilizado, como advim de um reprodutor de alto valor zootécnico ou ter sido submetido à criopreservação, assim como for utilizado em égua com endometrite persistentes póscobertura (Mccue, 2003). Para contemplar a múltipla ovulação na espécie equina tem sido frequentemente utilizado os análogos GnRH que mostram-se eficientes (Finan et al., 2016), pelo fato de estimularem a liberação do hormônio luteinizante (LH) e do hormônio folículo estimulante (FSH) a partir da glândula hipófise anterior (Campbell, 2012). Esses análogos estão totalmente associados às 
biotécnicas reprodutivas como a transferência de embriões (TE), utilizando-se como agente sincronizador de doadoras com receptoras (McKinnon et al., 1988; McKinnon et al., 2011) e em protocolos de superovulação (Alvarenga et al., 2001; Niswender et al., 2003). Assim, o objetivo desse trabalho foi descrever o uso do acetato de histrelina na múltipla ovulação em égua.

\section{Material e métodos}

Todos os procedimentos experimentais foram realizados de acordo com o Conselho Nacional de Experimentação Animal (CONCEA), que foram aprovados na Comissão de Ética no Uso de Animais (CEUA) da Faculdade Pio Décimo (Número de Protocolo: 22/2018)

O trabalho foi conduzido na Central de Reprodução Rancho 3M, localizado no município de Nossa Senhora do Socorro, Sergipe, Brasil. Foi utilizada uma égua Quarto de Milha, com idade de cinco anos, com aproximadamente $450 \mathrm{~kg}$. A égua era mantida em regime extensivo, submetida à uma dieta composta por $6 \mathrm{Kg}$ de concentrado divididos em duas porções e oferta de volumoso e água ad libitum. A égua se encontrava em plena competência reprodutiva, com dois embriões transferidos antes da realização do protocolo e sem anormalidades no ciclo, possibilitando sua participação no estudo.

Antes da realização do protocolo foram acompanhados dois ciclos da égua (Tabela 1) procedendo as avaliações nos meses de dezembro de 2017 e janeiro de 2018, com o auxílio de aparelho digital de ultrassom SonoScape A5 ${ }^{\circledR}$, equipado com transdutor linear de 5,0 Megahertz.

Tabela 1. Acompanhamento da dinâmica folicular de égua em dois ciclos antecedentes ao protocolo

\begin{tabular}{|c|c|c|c|c|c|c|c|}
\hline Data & Ovário direito & Ovário esquerdo & Edema uterino & $\begin{array}{c}\text { Prostaglandina } \\
\text { F2 } \alpha\end{array}$ & $\begin{array}{c}\text { Indução de } \\
\text { ovulação }\end{array}$ & $\begin{array}{c}\text { Inseminação } \\
\text { artificial }\end{array}$ & $\begin{array}{c}\text { Transferência } \\
\text { de embrião }\end{array}$ \\
\hline $07 / 12 / 17$ & Poli - & $\mathrm{CL}$ & 0 & $\bar{X}$ & & & \\
\hline $12 / 12 / 17$ & $35 \mathrm{~mm}$ & Poli - & 3 & & & & \\
\hline $14 / 12 / 17$ & $42 \mathrm{~mm}$ & Poli + & 3 & & & & \\
\hline $15 / 12 / 17$ & $45 \mathrm{~mm}$ & Poli + & 3,5 & & $\mathrm{X}$ & & \\
\hline $16 / 12 / 17$ & $48 \mathrm{~mm}$ & Poli - & 1 & & & $\mathrm{X}$ & \\
\hline $23 / 12 / 17$ & & & & & & & $X$ \\
\hline $25 / 12 / 17$ & $\mathrm{CL}$ & Poli - & 0 & $\mathrm{X}$ & & & \\
\hline $30 / 12 / 17$ & $33 \mathrm{~mm}$ & Poli + & 2 & & & & \\
\hline $02 / 01 / 18$ & $44 \mathrm{~mm}$ & Poli + & 2,5 & & & & \\
\hline 03/01/18 & $46,5 \mathrm{~mm}$ & Poli - & 1,5 & & & & \\
\hline 04/01/18 & CL & Poli - & 0 & & & & \\
\hline
\end{tabular}

mm=Milímetros, Poli-=Ovário poli folicular com alguns folículos $<18 \mathrm{~mm}$, Poli+= Ovário poli folicular com alguns folículos $>18 \mathrm{~mm}$.

O fármaco escolhido para o protocolo foi o acetato de histrelina com apresentação (Strelin ${ }^{\circledR}$ ) administrando 40\% da dose utilizada para indução de ovulação (100 $\mu \mathrm{g})$ (Tabela 2).

Tabela 2. Frequência de aplicações do hormônio utilizado no protocolo de múltipla ovulação

\begin{tabular}{|c|c|c|c|c|}
\hline$\overline{\text { Data }}$ & Dose & Volume & Horário & Fármaco \\
\hline $12 / 01 / 18$ & $5 \mathrm{mg}$ & $1,0 \mathrm{~mL}$ & $07: 00$ & Dinoprost Trometamina \\
\hline \multirow{2}{*}{$13 / 01 / 18$} & \multirow{2}{*}{$100 \mu \mathrm{g}$} & $0,4 \mathrm{~mL}$ & $07: 00$ & \multirow{2}{*}{ Acetato de Histrelina } \\
\hline & & $0,4 \mathrm{~mL}$ & $19: 00$ & \\
\hline \multirow{2}{*}{$14 / 01 / 18$} & \multirow{2}{*}{$100 \mu \mathrm{g}$} & $0,4 \mathrm{~mL}$ & 07:00 & \multirow{2}{*}{ Acetato de Histrelina } \\
\hline & & $0,4 \mathrm{~mL}$ & $19: 00$ & \\
\hline \multirow{2}{*}{$15 / 01 / 18$} & \multirow{2}{*}{$100 \mu \mathrm{g}$} & $0,4 \mathrm{~mL}$ & $07: 00$ & \multirow{2}{*}{ Acetato de Histrelina } \\
\hline & & $0,4 \mathrm{~mL}$ & $19: 00$ & \\
\hline \multirow{2}{*}{$16 / 01 / 18$} & \multirow{2}{*}{$100 \mu \mathrm{g}$} & $0,4 \mathrm{~mL}$ & 07:00 & \multirow{2}{*}{ Acetato de Histrelina } \\
\hline & & $0,4 \mathrm{~mL}$ & $19: 00$ & \\
\hline $17 / 01 / 18$ & $100 \mu \mathrm{g}$ & $0,4 \mathrm{~mL}$ & 07:00 & Acetato de Histrelina \\
\hline $17 / 01 / 18$ & $250 \mu \mathrm{g}$ & $1,0 \mathrm{~mL}$ & $19: 00$ & Acetato de Histrelina \\
\hline
\end{tabular}


Um dia antes do início do protocolo (12/01/18) foi realizada avaliação ultrassonográfica e identificado três folículos com diâmetro > $15 \mathrm{~mm}$. Em seguida, foi administrada uma dose de prostaglandina (PGF2 $\alpha$ ) com o intuito de promover a lise do corpo lúteo formado no último ciclo, e então, o protocolo teve início no dia 13/01/18 quando na avaliação ultrassonográfica a égua apresentava três folículos $>18 \mathrm{~mm}$ de diâmetro. Após a avaliação foi realizada a primeira aplicação do acetato de histrelina $(100 \mu \mathrm{g})$. $\mathrm{O}$ protocolo durou cinco dias, sendo o último dia realizado apenas uma administração da dosagem para o protocolo às 07:00 e às 19:00 utilizou a dosagem de indução de ovulação $(250 \mu \mathrm{g})$, finalizando o protocolo. No dia subsequente ao término do protocolo, a égua foi avaliada, totalizando sete avaliações ultrassonográficas.

\section{Resultado e discussão}

A impossibilidade de enquadramento de um número maior de animais nesta pesquisa não favoreceu a utilização do mesmo protocolo de sincronização, considerando que havia acompanhamento reprodutivo prévio pelo técnico responsável da central de reprodução, tendo controle sobre o ciclo estral da égua. Segabinazzi et al. (2015) descrevem a utilização da PGF2 $\alpha$ antecedente à realização do protocolo de múltipla ovulação para a sincronização de cio, realizando duas aplicações com o intervalo de 14 dias.

Tabela 3. Descrição das avalições ultrassonográficas durante o protocolo de múltipla ovulação

\begin{tabular}{|c|c|c|c|c|c|c|c|}
\hline$\overline{\text { Dia }}$ & Data & Horário & Ovário direito & Ovário esquerdo & Edema uterino & Útero & Aplicação do hormônio \\
\hline $1^{\mathrm{o}}$ & $13 / 01 / 18$ & 07:00 & $\begin{array}{l}18,5 \mathrm{~mm} \\
19,1 \mathrm{~mm}\end{array}$ & $18,8 \mathrm{~mm}$ & 1 & Com tônus & Sim \\
\hline $2^{\circ}$ & $14 / 01 / 18$ & 07:00 & $\begin{array}{l}20,8 \mathrm{~mm} \\
22,8 \mathrm{~mm}\end{array}$ & $\begin{array}{l}21,7 \mathrm{~mm} \\
17,8 \mathrm{~mm}\end{array}$ & 1 & Com tônus & Sim \\
\hline $3^{\circ}$ & $15 / 01 / 18$ & $13: 00$ & $\begin{array}{l}27,4 \mathrm{~mm} \\
25,4 \mathrm{~mm}\end{array}$ & $\begin{array}{l}24,8 \mathrm{~mm} \\
19,3 \mathrm{~mm}\end{array}$ & 1 & Com tônus & Sim \\
\hline $4^{\circ}$ & $16 / 01 / 18$ & 07:00 & $\begin{array}{l}31,1 \mathrm{~mm} \\
23,9 \mathrm{~mm}\end{array}$ & $\begin{array}{l}28,1 \mathrm{~mm} \\
23,4 \mathrm{~mm}\end{array}$ & 2 & Relaxado & Sim \\
\hline $5^{\circ}$ & $17 / 01 / 18$ & 19:00 & $\begin{array}{l}35,5 \mathrm{~mm} \\
27,7 \mathrm{~mm}\end{array}$ & $30,6 \mathrm{~mm}$ & 3 + Fluido & Relaxado & Sim \\
\hline $6^{\circ}$ & $18 / 01 / 18$ & 07:00 & $\begin{array}{l}22,7 \mathrm{~mm} \\
39,9 \mathrm{~mm}\end{array}$ & $27,3 \mathrm{~mm}$ & 3 & Relaxado & Não \\
\hline $7^{\circ}$ & $19 / 01 / 18$ & $07: 00$ & $1 \mathrm{CL}$ & Poli - & 0 & Com tônus & Não \\
\hline
\end{tabular}

Poli-=Ovário poli folicular com alguns folículos $<18 \mathrm{~mm}$.

Recentemente, o acetato de histrelina tem conferido resultados satisfatórios na indução e a sincronização de ovulação na dose $0,25 \mathrm{mg}$ (Kiser et al., 2013). Apesar da utilização da mesma dose no animal do experimento, não foi observada ovulação. O seu uso ainda é recomendado no início da estação de monta, no momento que as concentrações plasmáticas de LH são inferiores. A utilização de outros indutores como o estrato de pituitária equina purificado (EPE) conferiu bons resultados, com 70 a $90 \%$ dos animais respondendo ao protocolo, com média de ovulação de 1,7 a 3,8 folículos por ciclo (Niswender et al., 2003). O presente estudo tem concordância com o que foi descrito pelo autor citado, visto que ao quarto dia do protocolo, a égua apresentou três folículos $>27 \mathrm{~mm}$ de diâmetro, como exposto na (Tabela 3). Salientando que no último dia apenas dois folículos permaneceram com o crescimento esperado, suficiente para responder à indução.

A indução com utilização de acetato de deslorelina $750 \mu \mathrm{g}$ e 1650 IU de gonadrotofina coriônica humana (hCG) por via intramuscular, quando observado dois ou mais folículos $\geq 35 \mathrm{~mm}$, ou apenas um $\geq 38 \mathrm{~mm}$ mostrou-se eficiente para a indução da múltipla ovulação em éguas (Segabinazzi et al., 2015). Finan et al. (2016) avaliando a eficiência na múltipla ovulação do Biorelease deslorelina e ovuplant verificaram que o uso dos dois fármacos diminui significativamente o tempo de ovulação em relação ao grupo controle. Porém, o uso desse fármaco sob a forma de implante, aumentou o tempo no retorno ao estro se o implante não for removido (Henderson et al., 2012). Em outro protocolo que avaliava a 
eficiência do acetato de buserelina ( $40 \mu \mathrm{g}$ ) quando os folículos têm pelo menos $45 \mathrm{~mm}$ de diâmetro e o edema uterino diminuído, mostrou-se capaz de induzir a ovulação (Miki et al., 2016). Ainda, segundo esses autores, não se obteve diferenças significativas entre taxas de concepção e a ocorrência de múltipla ovulação entre os grupos.

Foi realizado o protocolo para inseminação com sêmen congelado, e após 36 horas a égua ainda não havia ovulado e apresentava fluido uterino, então, foi avaliada às 42 horas ainda não havendo ovulação, sendo que, dos dois folículos induzidos, um deles evoluiu e apresentava diâmetro de 27,3 mm (Figura 1). Após 42 duas horas foi optado por não realizar a inseminação pelo fato da égua apresentar edema uterino 3.

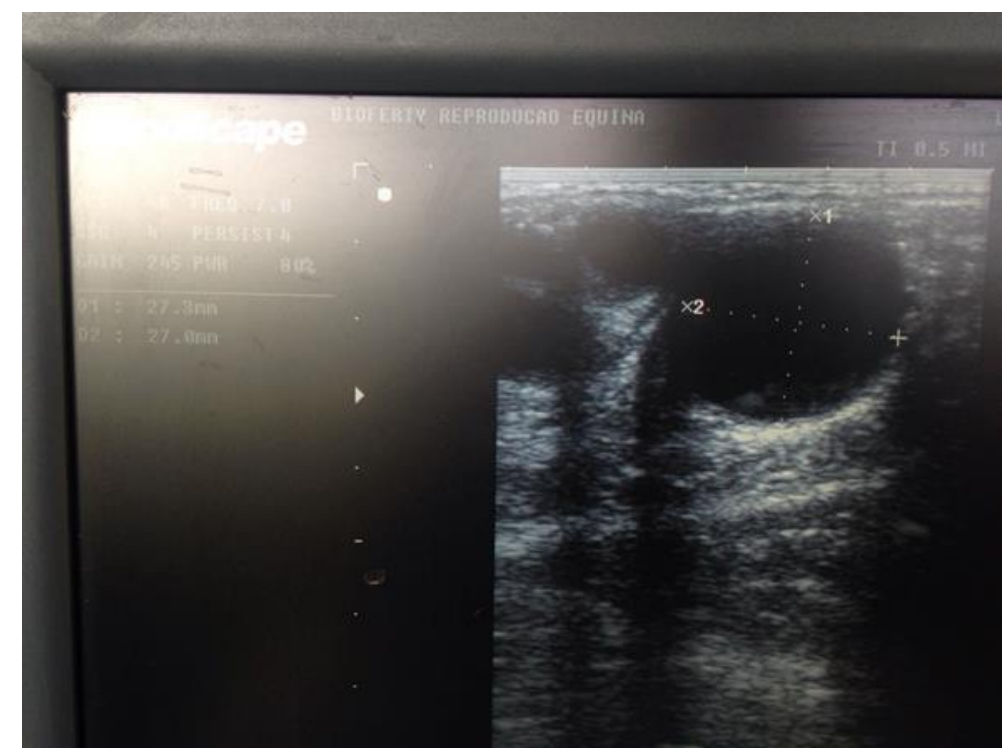

Figura 1. Folículo em atresia apresentando $\pm 27,3 \mathrm{~mm}$ de diâmetro.

O aumento dos níveis de estrógeno culminou na falha da ovulação pelo fato da dosagem da histrelina utilizada no protocolo de múltipla ovulação ser administrada de forma contínua durante o experimento, e desencadear o aumento do estrógeno circulante, resultando na diminuição nos níveis de LH e consequentemente não promover a ovulação. A eficiência na indução de ovulações duplas é relacionada ao momento do início do tratamento; melhores taxas são observadas quando o protocolo com deslorelina iniciou com no mínimo dois folículos entre 20 e $25 \mathrm{~mm}$, contudo, no protocolo utilizado, administrouse a dose com apenas um folículo apresentando $45 \mathrm{~mm}$ de diâmetro (Nagao et al., 2012). Existem trabalhos que avaliaram a contribuição do acetato de histrelina e o acetato de deslorelina, este último, também muito utilizado em substituição ao primeiro (Lindholm et al., 2011).

Outro trabalho concorda como o exposto por (Nagao et al., 2012) que confirma também a importância do momento ideal para o início do tratamento super ovulatório, verificando melhores respostas quando o folículo atingiu o diâmetro médio entre 20-23 mm.

\section{Conclusão}

Conclui-se que a égua utilizada não respondeu ao protocolo, não evidenciando múltiplas ovulações. Apesar do resultado obtido, já se conhece a eficiência farmacológica do acetato de histrelina na múltipla ovulação.

\section{Referências bibliográficas}

Alvarenga, M. A., McCue, P. M., Bruemmer, J., Neves Neto, J. R. \& Squires, E. L. (2001). Ovarian superstimulatory response and embryo production in mares treated with equine pituitary extract twice daily. Theriogenology, 56(5):879-887.

Campbell, M. (2012). It's all in the timing: ovulation induction in the mare. Veterinary Record, 170538539. 
Finan, S. A., Lamkin, E. L. \& McKinnon, A. O. (2016). Comparative efficacy of BioRelease Deslorelin ${ }^{\circledR}$ injection for induction of ovulation in oestrus mares: a field study. Australian Veterinary Journal, 94(9):338-340.

Ginther, O. J., Beg, M. A., Donadeu, F. X. \& Bergfelt, D. R. (2003). Mechanism of follicle deviation in monovular farm species. Animal Reproduction Science, 78(3):239-257.

Ginther, O. J., Whitmore, H. L. \& Squires, E. L. (1972). Characteristics of estrus, diestrus, and ovulation in mares and effects of season and nursing. American Journal of Veterinary Research, 33(10):19351939.

Henderson, I. S. F., Brama, P., Osborne, M. \& Beltman, M. E. (2012). Interovulatory intervals in mares receiving deslorelin implants in Ireland (2009 to 2010). Veterinary Record, 170(21):541-541.

Kiser, A. M., Sudderth, A. K., Brinsko, S. P., Burns, P. J., Douglas, R. H. \& Blanchard, T. L. (2013). Comparison of efficacy of two dose rates of histrelin for inducing ovulation in broodmares. Journal of Equine Veterinary Science, 33(10):820-822.

Lindholm, A. R. G., Ferris, R. A., Scofield, D. B. \& McCue, P. M. (2011). Comparison of deslorelin and histrelin for induction of ovulation in mares. Journal of Equine Veterinary Science, 5(31):312313.

Loy, R. G. \& Hughes, J. P. (1966). The effects of human chorionic gonadotrophin on ovulation, length of estrus, and fertility in the mare. The Cornell Veterinarian, 56(1):41-50.

Mccue, P. M. (2003). Induction of ovulation. In N. E. Rpbinson (Ed.), Current Therapy in Equine Medicine (pp. 240-242.). Philadelphia, USA: Saunders.

McKinnon, A. O., Squires, E. L., Harrison, L. A., Blach, E. L. \& Shideler, R. K. (1988). Ultrasonographic studies on the reproductive tract of mares after parturition: effect of involution and uterine fluid on pregnancy rates in mares with normal and delayed first postpartum ovulatory cycles. Journal of the American Veterinary Medical Association, 192(3):350-353.

McKinnon, A. O., Squires, E. L., Vaala, W. E. \& Varner, D. D. (2011). Equine Reproduction. USDA: John Wiley \& Sons.

Miki, W., Oniyama, H., Takeda, N., Kimura, Y., Haneda, S., Matsui, M., . . Nambo, Y. (2016). Effects of a single use of the GnRH analog buserelin on the induction of ovulation and endocrine profiles in heavy draft mares. Journal of Equine Science, 27(4):149-156.

Nagao, J. F., Neto, J. R. N., Papa, F. O., Alvarenga, M. A., Freitas-Dell'Aqua, C. P. \& Dell'Aqua Junior, J. A. (2012). Induction of double ovulation in mares using deslorelin acetate. Animal Reproduction Science, 136(1-2):69-73.

Niswender, K. D., Alvarenga, M. A., McCue, P. M., Hardy, Q. P. \& Squires, E. L. (2003). Superovulation in cycling mares using equine follicle stimulating hormone (eFSH). Journal of Equine Veterinary Science, 23497-500.

Segabinazzi, L. G., Steigleder, L. F., Kaipper, R., Rodriigues, T. S., Aragão, O., Dell'aqua Júnior, Antonio \& Alvarenga, M. A. (2015). Indução de múltiplas ovulações em éguas com baixas doses de acetato de deslorelina sem acompanhamento folicular prévio. Enciclopérida Biosfera, 11(14881494).

Woods, J., Bergfelt, D. R. \& Ginther, O. J. (1990). Effects of time of insemination relative to ovulation on pregnancy rate and embryonic-loss rate in mares. Equine Veterinary Journal, 22(6):410-415.

Recebido: 20 março, 2019

Aprovado: 16 abril, 2019.

Publicado: 30 abril, 2019.

Licenciamento: Este artigo é publicado na modalidade Acesso Aberto sob a licença Creative Commons Atribuição 4.0 (CC-BY 4.0), a qual permite uso irrestrito, distribuição, reprodução em qualquer meio, desde que o autor e a fonte sejam devidamente creditados. 